\title{
Topiramate Reduces Aortic Cross-Clamping-Induced Lung Injury in Male Rats
}

\author{
Aysel Kurt ${ }^{1}$, Yildiray Kalkan², Hasan Turut ${ }^{3}$, Medine Cumhur Cure ${ }^{4}$, Levent Tumkaya ${ }^{5}$, Erkan Cure,*
}

\begin{abstract}
Background: Topiramate (TPM) decreases cytokine release and generation of reactive oxygen species (ROS).

Cytokine and endothelin-1 (ET-1) secretion and ROS formation play an important role in ischemia-reperfusion (I/R) injury. We aimed to evaluate whether TPM prevents damage occurring in lung tissue during I/R.

Materials and Methods: A total of 27 Wistar albino rats were divided into three groups of nine. To the I/R group, two hours of ischemia via infrarenal abdominal aorta cross-ligation and then two hours of reperfusion process were applied. TPM (100 mg/kg/day) orally for seven days was administered in the TPM treatment group. After the last dose of TPM treatment, respectively, two hours of ischemia and two hours of reperfusion were applied in this group.

Results: Tumor necrosis factor-alpha (TNF-a) $(p<0.05)$, malondialdehyde (MDA) $(p<0.05)$, myeloperoxidase $(M P O)(p<0.05)$ and ET-1 $(p<0.05)$ levels of TPM treatment group's lung tissue were significantly lower than for the I/R group. Caspase-3 and histopathological damage were rather lower than that of the I/R group.

Conclusions: During I/R, lung damage occurs due to excessive TNF-a and ET-1 release and ROS generation. TPM could well reduce development of lung damage by decreasing cytokine and ET-1 release and levels of ROS produced.
\end{abstract}

\section{KEYWORDS}

Topiramate; ischemia-reperfusion; lung injury; tumor necrosis factor-alpha; endothelin-1

\section{AUTHOR AFFILIATIONS}

${ }^{1}$ Private Practice, Department of Thoracic Surgery, Rize, Turkey

2 Private Practice, Department of Histology and Embryology, Rize, Turkey

${ }^{3}$ Recep Tayyip Erdogan University, School of Medicine, Department of Thoracic Surgery, Rize, Turkey

${ }^{4}$ Istanbul Laboratory, Department of Biochemistry, Istanbul, Turkey

${ }^{5}$ Recep Tayyip Erdogan University, School of Medicine, Department of Histology and Embryology, Rize, Turkey

${ }^{6}$ Camlica Erdem Hospital, Department of Internal Medicine, Istanbul, Turkey

* Corresponding author: Camlica Erdem Hospital, Department of Internal Medicine, Uskudar, Istanbul, Turkey; e-mail: erkancure@yahoo.com

Received: 10 February 2018

Accepted: 2 November 2018

Published online: 22 January 2019

Acta Medica (Hradec Králové) 2018; 61(4): 144-149

https://doi.org/10.14712/18059694.2018.133

(c) 2018 The Authors. This is an open-access article distributed under the terms of the Creative Commons Attribution License (http://creativecommons.org/licenses/by/4.0), which permits unrestricted use, distribution, and reproduction in any medium, provided the original author and source are credited. 


\section{INTRODUCTION}

Pulmonary dysfunction is one of the problematic conditions developing after ischemic reperfusion (I/R) on account of lung transplantation (1), extracorporeal circulation operations (2, 3) after resuscitation for circulatory arrest (4) or severe hemorrhagic shock (5). Reperfusion of ischemic tissue causes microvascular dysfunction and parenchymal cells, depending on local and systemic responses. Lung damage developing during the I/R process has been shown to be associated with physiological, biochemical and histopathological changes. Lung I/R injury is described by neutrophil extravasation, interstitial edema, disruption of epithelial integrity, and leakage of protein into the alveolar space, all of which are associated with severe alterations in gas exchange $(6,7)$. In its early stages, activated alveolar macrophages and pro-inflammatory cytokines such as tumor necrosis factor-alpha (TNF-a) increase, and reactive oxygen radicals (ROS) and later, in last stages of perfusion, activated neutrophils play crucial roles in this uncontrolled inflammation process $(8,9)$.

In showing the lung damage of the I/R process, malondialdehyde (MDA), a marker for lipid peroxidation and oxidative stress, and myeloperoxidase (MPO), a marker for activation of neutrophils located on the lung tissue, are both valuable and reliable parameters (10). Besides, endothelin-1 (ET-1), released from damaged endothelial cells, is another effective mediator causing vascular inflammation, cell proliferation, and fibrosis. Additionally, this ET-1 plays a crucial role in the development of acute lung injury (11).

Topiramate (TPM) is an agent used for epilepsy and migraine treatment. It inhibits voltage-gated sodium and calcium channels, blocks glutamate a-amino-3-hydroxy-5-methyl4-isoxazole propionic acid (AMPA)/kainate receptors and enhances gamma-aminobutyric acid (GABA), a receptor-mediated chloride (12). TPM has also been shown to have antioxidant effects, reducing TNF-a release and oxidative stress (13). In addition, decreases in the level of MDA enzyme has been reported in other studies (14). Another positive effect of TPM is diminished insulin resistance (15).

In this study, we aimed to evaluate the effects of TPM on ET-1, TNF-a, MDA and MPO enzyme activity which play crucial roles in producing ROS, to assess apoptosis via measuring caspase- 3 enzyme activity and the effect of TPM on it, and to determine whether TPM has a preventive effect on I/R lung damage.

\section{MATERIALS AND METHODS}

Animals used in the experimental study were 12-15-week-old experimental design male Wistar albino rats $(n=27)$ weighing 250-300 g. The study design was approved by license no: $2014 / 9$ by the local ethical committee at the University and all protocols conformed to the Guide for the Care and Use of Laboratory Animals (NIH, 1985). Rats were kept under standard temperature and environment with a 12-h light-dark cycle and were given food and water ad libitum. The rats were randomly divided into three groups as follows.

Group I: Control groups ( $n=9)$, only received saline solution intraperitoneally. They underwent a midline laparotomy, with the infrarenal abdominal aorta (IAA) being dissected and crossed without obstruction. Group II: I/R groups $(n=9)$, after the midline laparotomy of rats, the IAA was clamped for 120 mins, followed by 120 mins of reperfusion. Group III: For I/R+TPM groups ( $\mathrm{n}=9)$, TPM $\left(100 \mathrm{mg} / \mathrm{kg} /\right.$ day, Topamax ${ }^{\circledR}$, Johnson and Johnson) was orally (via gastric gavage) given for seven sequential days prior to I/R. After seven days' administered TPM, this group underwent respectively 120 mins of ischemia and 120 mins of reperfusion.

In humans, oral dose of TPM has been found to be effective within 2 hours in plasma and at target dose within 4 weeks (16). We chose $100 \mathrm{mg} / \mathrm{kg} /$ day of TPM and for 1 week since the previous animal studies used this dose and duration (17-19).

\section{PROCEDURE OF AORTIC ISCHEMIA-REPERFUSION}

The rats were anesthetized by intraperitoneal injection of $50 \mathrm{mg} / \mathrm{kg}$ ketamine hydrochloride (Ketalar; Eczacibasi, Istanbul, Turkey) and were maintained by intramuscular injections of $25 \mathrm{mg} / \mathrm{kg}$ ketamine hydrochloride when needed for all groups. The surgery was performed with rats placed in the supine position under a heating lamp. After a median laparotomy under sterile conditions, the IAA was explored by gentle dissection of the retroperitoneum, and then an atraumatic microvascular clamp was placed across the IAA for 120 mins of ischemia. After the abdomen incision was closed, the wound was covered with plastic wrap to prevent the loss of heat and fluid. Ten milliliters of normal saline was dribbled into the peritoneal cavity to maintain fluid balance. The traumatic microvascular clamp was removed on the IAA and lower extremities were reperfused for 120 mins. Aortic occlusion and reperfusion were verified by observing the loss and reappearance of the pulsation on the distal aorta. The I/R model was designed according to a method similar to previous studies (20). After ending the procedure, the rats were sacrificed under anesthesia and then lung tissues were obtained for biochemical and histological analyses.

\section{TISSUE HOMOGENATES}

The lung tissue samples were homogenized in Phosphate Buffer Saline (PBS) (50 mM, pH 7.4) and centrifuged at $10,000 \mathrm{~g}$ for 20 mins. The supernatant was removed to be aliquoted into tubes. It was kept frozen at $-80{ }^{\circ} \mathrm{C}$ and biochemical parameters such as TNF-a, MDA, MPO, ET-1 were studied within a month.

\section{MEASUREMENT OF PROTEIN}

Tissue homogenate protein assay is a Lowry protocol based on the Biuret reaction. The peptide bonds of proteins react with copper under alkaline conditions to produce $\mathrm{Cu}+$, which reacts with the Folin reagent, and the Folin-Ciocalteau reaction (21).

\section{TISSUE TNF-a}

The TNF-a levels were measured using an enzyme-linked immunosorbent assay (ELISA) method. We used a commercially available rat TNF-a ELISA kit (eBioscience, Vienna, Austria). The method was according to the manufacturer's instructions. Absorbance was measured at a wavelength of $450 \mathrm{~nm}$ 
with an ELISA reader. The TNF-a levels are given as $\mathrm{pg} / \mathrm{ml}$. The intra-assay and inter-assay coefficient of variation was $<5 \%$ and $<10 \%$, respectively. The limit of detection (LOD) for the TNF-a assay was $11 \mathrm{pg} / \mathrm{ml}$.

\section{TISSUE MDA}

MDA levels were measured using the double heating method of Draper and Hadley. The principle of the method is the spectrophotometric measurement of the color generated by the reaction of thiobarbituric acid (TBA) by MDA. The MDA levels are given as $\mu \mathrm{mol} / \mathrm{L}(22)$.

\section{TISSUE ET-1}

The concentration of ET-1 was measured by the ELISA method. We used a commercially available rat ET-1 ELISA kit (Elabscience, Wuhan, P.R.C) in this study. The procedure for the ELISA method was applied according to the manufacturer's instructions. Absorbance was measured at a wavelength of $450 \eta \mathrm{m}$ by an ELISA reader. The levels of ET-1 are presented as $\mathrm{pg} / \mathrm{ml}$ and the coefficient of variation was $<10 \%$. The minimum detectable dose of the ET-1 test was $4.69 \mathrm{pg} / \mathrm{ml}$.

\section{TISSUE MPO}

The levels of MPO were measured using the ELISA method. We used a commercially available rat MPO ELISA kit (Elabscience, Wuhan, P.R.C) in the study. Absorbance was measured at a wavelength of $450 \mathrm{~nm}$ with the ELISA reader. The levels of MPO are presented as $\mathrm{ng} / \mathrm{ml}$. The coefficient of variation was $<10 \%$. The minimum detectable dose of MPO test was $0.19 \mathrm{ng} / \mathrm{ml}$ in the method. When dividing the values we achieved by protein levels, the final results were expressed as $\mu \mathrm{mol} / \mathrm{g}$ protein.

\section{IMMUNOHISTOLOGICAL EVALUATION}

In immunohistochemical staining, 3- $\mu \mathrm{m}$ thick sections of the lung tissues were cut off and allowed to stay in xylene for 20 mins before the implementation of an alcohol series (50-100\%), and afterward allowed to stay for 30 mins in an $\mathrm{H}_{2} \mathrm{O}_{2}$ solution. After being washed with PBS, these portions were heated in citrate buffer solution at $800 \mathrm{~W}$ for 5 mins (twice) and allowed to remain in a secondary blocker substance for 30 mins. Each slide was allowed to stand for 60 mins in different dilutions of the primary antibody (Anti-Caspase 3 antibody (ab4051), Abcam plc, Cambridge CB4 OFL UK). A diaminobenzidine (DAB Substrate Kit, ab64238, Abcam plc, Cambridge CB4 OFL UK) solution was used as a chromogen, with Mayer's hematoxylin as a counterstain for 3-5 mins, and PBS as a negative control. The preparations were photographed after being covered with the eligible covering materials. Histopathological examinations were evaluated according to the previous studies models (23-25). Consequently, for immunohistochemical staining, the preparations were divided into four categories according to the tissue percentage of immunopositive reaction areas: mild $(+)$, moderate $(++)$, severe $(+++)$ and very severe $(++++)$. The blocked tissues were cut into $4-\mu \mathrm{m}$ thick sections before being stained with hematoxylin and eo$\sin (H \& E)$ and Masson's trichrome, and then the fields found to be suitable (total 150 zones) for histopathological assessment were photographed. The tissues were appraised with a blindly graded exam in groups by two expert histologists.

\section{STATISTICAL ANALYSES}

Continuous variables were reported as the mean \pm standard deviation and statistical analysis was performed using SPSS for Windows (SPSS, USA) version 17. Comparison of the groups' biochemical parameters such as TNF-a, MDA, MPO, ET-1 were performed using the one-way ANOVA and Bonferroni post hoc tests. For histopathological parameters, the Mann-Whitney $U$ test was used to compare the groups and differences were considered significant at $p<0.05$.

\section{RESULTS}

TNF-a, MDA, MPO and ET-1 levels of the I/R group were found significantly higher than the controls and TPM-treatment group. In the TPM-treatment group, TNF- $a$, MDA, MPO and ET-1 levels were lower compared with the I/R group's levels. TNF- $a$, MPO and ET-1 levels of TPM-treatment group was similar to the control group, while MDA levels were slightly higher than of controls. All biochemical parameters are given in Table 1.

Caspase-3 enzyme activity in the I/R group was significantly higher than both in control and TPM-treatment groups. The I/R group had severe histopathological damage. In the TPM treatment group, caspase-3 activity was lower than for the I/R group and also histopathological damage to lung tissue was less extent. All histopathological results are providedin Table 2.

Tab. 1 All biochemical results of the three groups.

\begin{tabular}{|l|l|l|l|}
\hline & I/R & I/R+TPM & Control \\
\hline TNF-a pg/mg & $197.3 \pm 26.6 \chi^{*}$ & $159.9 \pm 40.9$ & $140.3 \pm 27.8$ \\
\hline MDA $\mathbf{n m o l} / \mathbf{m g}$ & $2.96 \pm 0.34^{*} \#$ & $2.54 \pm 0.36^{* *}$ & $2.11 \pm 0.42$ \\
\hline MPO $\mathbf{n g} / \mathbf{m g}$ & $0.60 \pm 0.16^{* *} \#$ & $0.45 \pm 0.10$ & $0.43 \pm 0.08$ \\
\hline ET-1 $\mathbf{p g} / \mathbf{m g}$ & $8.6 \pm 2.3^{*} \mathrm{w}$ & $5.5 \pm 1.7$ & $4.7 \pm 1.6$ \\
\hline
\end{tabular}

Abbreviations: I/R: Ischemia reperfusion; I/R+TPM: Ischemia reperfusion + Topiramate; TNF-a, Tumor necrosis factor-alpha; MDA, malondialdehyde; MPO, myeloperoxidase; ET-1, Endothelin-1.

${ }^{*} \mathrm{p}<0.001, \mathrm{xp}=0.003,{ }^{* *} \mathrm{p}<0.05 \mathrm{vs}$. control group $w p=0.004, \# p<0.05$ vs. I/R+TPM group

Tab. 2 Histopathologic examination of lung tissue.

\begin{tabular}{|l|l|l|l|}
\hline & Control & I/R & I/R+TPM \\
\hline Inflammation & $0.00 \pm 0.35$ & $3.00 \pm 0.71^{*} \mathbb{~}$ & $2.00 \pm 0.46^{*}$ \\
\hline Congestion & $0.00 \pm 0.35$ & $1.50 \pm 0.53^{*}$ & $1.50 \pm 0.53^{*}$ \\
\hline Edema & $0.00 \pm 0.35$ & $3.00 \pm 0.46^{*}$ & $1.50 \pm 0.53^{*}$ \\
\hline $\begin{array}{l}\text { Vacuolization } \\
\text { Epithelial degeneration }\end{array}$ & $0.00 \pm 0.35$ & $3.00 \pm 0.64^{*} ¥$ & $2.00 \pm 0.52^{*}$ \\
\hline $\begin{array}{l}\text { Alveolar macrophages } \\
\text { density }\end{array}$ & $0.00 \pm 0.46$ & $3.50 \pm 0.53^{*} \mathbb{q}$ & $1.50 \pm 0.53^{*}$ \\
\hline Caspase-3 & $1.00 \pm 0.64$ & $3.00 \pm 0.74^{*} \pi$ & $3.50 \pm 0.89^{*}$ \\
\hline
\end{tabular}

Abbreviations: I/R, Ischemia reperfusion; I/R+TPM, Ischemia reperfusion + Topiramate

* $p<0.001$ vs. control group

|| $p<0.001, ¥ p=0.002, \pi p=0.005$ vs. I/R+TPM group 
The histopathological examination for the control group of rat lungs indicated that the tissue integrity was not damaged, and possessed normal healthy histological morphology. This interstitial space of tissues was not occupied by polymorph nuclear leukocyte (PMNL) cells and was not observed to have congestion. In particular, despite the fact that there was mild epithelial cell 8 debris in terminal bronchioles, the integrity of all structures was intact, and lamina propria in the structures were found to be of normal morphology. In addition, the number of macrophages in the alveoli was normal, and a mild pigmentation in the macrophages was observed (Figure 1A).

Histopathological examination of the lung tissues of the I/R group showed that interstitial sites were locally expanded in sites where the transition areas of respiratory bronchioles, and edema and hyaline casts structures were observed in this areas. Moreover, dilatation of saccusalveolaris, loss of the normal structure in some saccusalveolaris, heavy injury in alveolar epithelial cells and PMNL infiltration of the surrounding areas BALT were observed in this group. In addition to edema in the subepithelial bronchial area, irregularity in the lamina propria and fibroblastic focusing in these areas were seen (Figure 1B). The number of alveolar macrophages was increased, their sizes were weighted, they were densely stained with eosinophilia and their pigmentations were shown as highly prominent. In the lung tissues of the I/R group, which had no healthy normal histological features, there was a significantly higher number of inflammatory cells in interstitial sites and more congestion found in small capillary vessels were significantly greater than that of controls and I/R+TPM treatment groups. Mild pigmentations were observed around bronchi and terminal bronchioles in the lungs of the I/R group. Some of the rats had mild perivascular edema, focal interstitial, intra-alveolar mononuclear leukocyte ( $M N L)$ infiltration and mild hyaline cast-like proteinaceous exudate.

Histopathological examination of lung tissue in the I/R+TPM group showed that congestion of the small vessels was reduced more compared with the I/R group, and although it did not entirely disappear, it was observed to be similar to the control group. The density of fibrosis was detected in the fibroblastic focusing areas located in the interstitial sites, and hyaline cast-structures and edema were decreased. Epithelial cell debris was noted in the bronchi 9 and bronchioles, and PMNL infiltrations developed in the lamina propria were decreased in this group. In particular, degeneration of epithelial cells in the alveoli in I/R group lungs, epithelial cell debris, increased interstitial connective tissue presence and density of alveolar macrophages were observed significantly reduced in this group (63\%) (Figure $1 C$ ). In this group, pigmentation areas was detected around the bronchial vessels and near the terminal bronchioles, and both densities of these and their size were found significantly decreased compared to the I/R group. The number of alveolar macrophages increased when compared with the control group, but pigmentation was similar to the controls. This difference was found to be statistically different $(p<0.05)$.

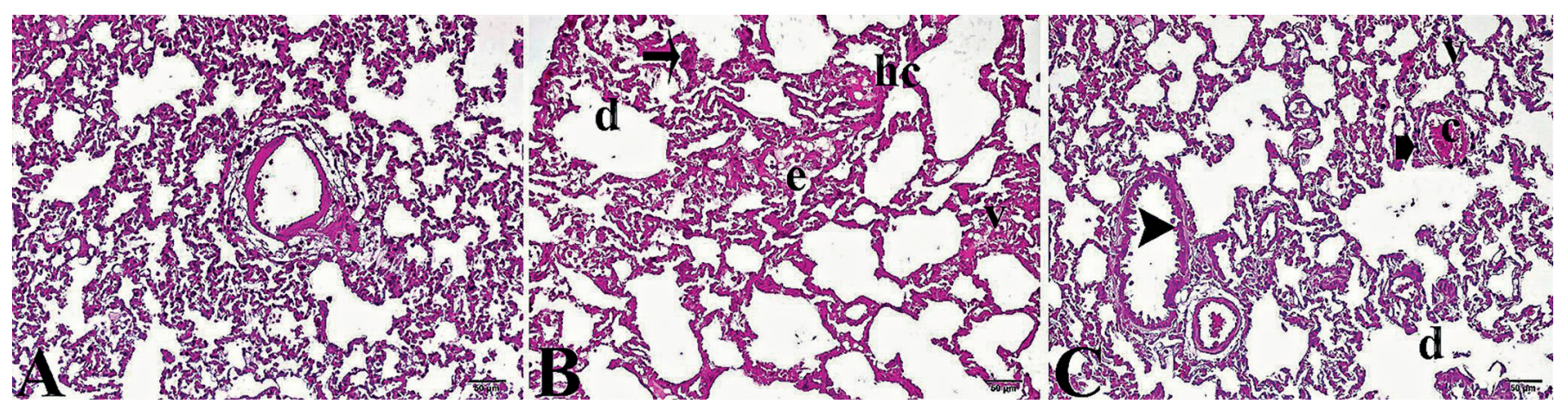

Fig. 1 Histopathological examination of lung tissue stained by hematoxylin-eosin stain by light microscopy. A: Normal lung tissue architecture in control group. B: I/R group, v: vacuolization, e: edema, d: alveolar dilatation, c: congestion, arrow: mononuclear cell infiltration, hc: hyaline cast structure. C: I/R+TPM group, v: vacuolization, c: congestion, arrowhead: degenerating epithelial cells, short arrow: pigmentation, $d$ : decreased alveolar dilatation, I/R: ischemia reperfusion, I/R+TPM: ischemia reperfusion + Topiramate.

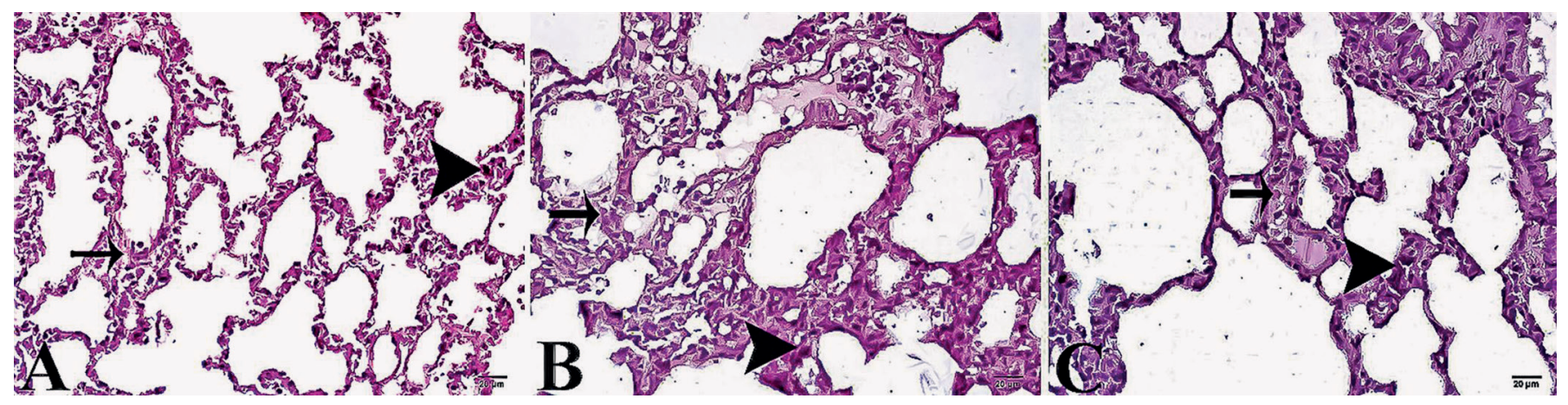

Fig. 2 Histopathological examination of lung tissue stained by immunoperoxidase method by light microscopy. A: control group. B: I/R group. C: I-R+TPM, arrowhead: strong positivity, arrow: weak positivity, immunoperoxidase stained Anti-Caspase-3 antibody. I/R: ischemia reperfusion, I/R+TPM: ischemia reperfusion + Topiramate. 


\section{DISCUSSION}

According to the results of our study, there was severe histopathological damage in the I/R group. Lung tissue was prominently damaged. Caspase-3 enzyme activity was found to be significantly increased. On the other hand, biochemical examination of lung tissue determined that TNF-a, MDA, MPO, and ET-1 levels were higher. According to histopathological examination, the TPM-treatment group had less lung tissue damage compared to the I/R group. Furthermore, TNF-a, MDA, MPO and ET-1 levels were found significantly lower than for the I/R group.

During I/R process, severe neutrophil and lymphocyte migration occur to the lungs and as a consequence, dense degranulation from leukocytes and severe development of ROS occurs, with increased proteases causing endothelial dysfunction (26). Thus, pulmonary capillary permeability increases, and interstitial edema develops (7). Over the reperfusion process, neutrophil adherence to the endothelial cells releases proteolytic enzymes such as MPO and thus increases ROS production, and activation of caspase- 3 enzyme systems all damage the lung tissues $(10,27)$. In our study, both MDA and MPO levels were significantly higher than that of both control and TPM-treatment groups. Increased MDA levels, the end product of lipid peroxidation, illustrates the increased ROS production throughout the I/R process. ROS development can lead to endothelial dysfunction and lung damage. MPO converts chloride ions and peroxides to hypochlorous acid during the I/R, and this acid is one the most potent oxidants, leading to lung tissue damage (10). In our study, increased MPO levels indicate indirectly the excessive ROS formation in the lung tissue.

TPM has been reported to decrease lipid peroxidation and MDA is an end product of lipid peroxidation $(14,19,28)$. At the same time, it has been shown that TPM decreases ROS generation via increasing the levels of antioxidant enzymes such as superoxide dismutase, catalase, and glutathione peroxidase during oxidative stress (17). During I/R, both increases in catabolism of nitric oxide (NO) and NO-dependent vasodilation of vessels lead to vessel damage (26). MPO enzyme uses NO as a substrate and then it increases NO catabolism (29). Thus, increased MPO enzyme activity indirectly indicates a decrease in NO level. Also, TPM increases NO release by inducing the NO synthase enzyme (30). Because of decreased NO levels, the production of asymmetric dimethyl arginine is increased, and this causes endothelial dysfunction. On the other hand, it has been reported that TPM decreases I/R damage via regulation of NO release (31). Our study showed that both MDA and MPO enzyme activity in the TPM-treatment group were significantly lower compared to the I/R group. Increased MPO level in the IR group may cause NO level to decrease and cause lung injury. TPM might have reduced lipid peroxidation, releasing MPO enzymes from neutrophils and monocytes and leading to ROS production during the I/R processin the TPM-treatment group.

During I/R, there occurs significant TNF-a release (32) and this leads the leukocytes to adhere to endothelial tissue. Thus, MPO and other lytic enzymes are released from leucocytes. Additionally, ROS production, synthesis of prostaglandins, platelet-activating factor (PAF), and thromboxane A2 (TXA2) release also result from TNF-a induction. Release of these substrates strengthens vasoconstriction even more (7). Both TNF- $a$ and the other pro-inflammatory cytokines released because of TNF- $a$ and ROS produced induce apoptosis via caspase enzyme pathway activation and thus lead to lung tissue damage (6). I/R studies have shown that blocking of TNF-a limits the lung damage (33). TPM has been shown to decrease I/R damage blocking release of TNF- $a$ and other cytokines $(31,34)$. Our study, similar to this, showed that significant TNF-a release was found in the I/R group, whilst the TPM treatment group had restricted TNF-a release.

ET-1 causes severe vasoconstriction via binding toits receptors located on the vascular smooth muscle cells (11). Increased ET-1 levels are known to lead to pulmonary hypertension (35), cardiac failure (36), and bronchial asthma (37). The pulmonary system's vascular smooth muscle cells and the other non-vascular organs' (such as trachea and bronchial) tissues' smooth muscle cells are affected by ET-1, which then leads to a potent construction with long-term duration, resulting in pulmonary vascular vasoconstriction and bronchoconstriction. This pulmonary effect of ET-1 has been reported to function via entering of calcium into the cells alongside increased leukotriene, TXA2 and PAF release $(38,39)$. Significant cytokine release and ROS production lead to endothelial dysfunction, while release of ET-1 increases due to ET-1 gene expression (39). During the I/R process, ET-1 release has been reported to increase cell damage, while blocking ET-1 release reduces tissue damage $(11,38-41)$. Additionally, it is known that NO-released from endothelial tissue is broken down because of endothelial dysfunction and severe tissue damage can occur due to imbalance of the NO/ET-1 ratio. In our study, ET-1 levels were profoundly higher in the I/R group and prominently lower in the TPM-treatment group. TPM could well protect endothelial tissue, decreasing levels of cytokines released and ROS produced and thus lead to preventing increasing ET-1 release.

During the I/R process, significant cytokine release and ROS production can lead to apoptosis, potentially resulting in tissue damage $(6,40)$. In a recent study, the I/R group's caspase- 3 enzyme activity was found as profoundly increased and lung tissue was severely damaged. TPM is known to be a suppressor of caspase-3 enzyme activity (42). In our study, the TPM-treatment group's caspase- 3 enzyme activity was prominently lower than that of the I/R group. TPM could very well prevent induction of apoptosis and thus decrease lung damage.

Lung tissue, due to its high sensitivity to injury, is damaged by cytokines and ROS production emerging during the abdominal I/R process. Increased ET-1 level leads to increased ROS and endothelial dysfunction. Release of MPO enzymes by leukocytes in damaged tissue not only causes an increase in ROS levels but also utilizes NO as a substrate, consuming the NO. Consequently, increased ROS may damages NO-dependent vasodilation and because NO levels are consumed by MPO, lung damage increases even more than before. TPM could highly prevent the lung from damage by decreasing caspase- 3 enzyme activity, cytokine and ET-1 release and ROS levels generated. 


\section{REFERENCES}

1. Hatachi G, Tsuchiya T, Miyazaki T, et al. The poly (adenosine diphosphate-ribose) polymerase inhibitor PJ34 reduces pulmonary ischemia reperfusion injury in rats. Transplantation 2014; 98: 618-24.

2. Ng CS, Wan S, Yim AP, Arifi AA. Pulmonary dysfunction after cardiac surgery. Chest 2002; 121: 1269-77.

3. Cao QF, Qu MJ, Yang WQ, Wang DP, Zhang MH, Di SB. Ischemia postconditioning preventing lung ischemia-reperfusion injury. Gene 2015; 554: $120-4$.

4. Shimamoto A, Pohlman TH, Shomura S, Tarukawa T, Takao M, Shimpo $\mathrm{H}$. Toll-like receptor 4 mediates lung ischemia-reperfusion injury. Ann Thorac Surg 2006; 82: 2017-23.

5. Reino DC, Pisarenko V, Palange D, et al. Trauma hemorrhagic shock-induced lung injury involves a gut-lymph-induced TLR4 pathway in mice. PLoS One 2011; 6: e14829.

6. Tang PS, Mura M, Seth R, Liu M. Acute lung injury and cell death: how many ways can cells die? Am J Physiol Lung Cell Mol Physiol 2008; 294: 632-41.

7. Ng CS, Wan S, Arifi AA, Yim AP. Inflammatory response to pulmonary ischemia reperfusion injury. Surg Today 2006; 36: 205-14.

8. Jiang L, Li L, Shen J, Qi Z, Guo L. Effect of dexmedetomidine on lung ischemia-reperfusion injury. Mol Med Rep 2014; 9: 419-26.

9. Eppinger MJ, Deeb GM, Bolling SF, Ward PA. Mediators of ischemia-reperfusion injury of rat lung. Am J Pathol 1997; 150: 1773-84.

10. Türüt $H$, Kurutas $E B$, Bulbuloglu $E$, et al. Zinc aspartate alleviates lung injury induced by intestinal ischemia-reperfusion in rats. J Surg Res 2009; 151: 62-7.

11. Comellas AP, Briva A. Role of endothelin-1 in acute lung injury. Transl Res 2009; 153: 263-71.

12. White HS, Smith MD, Wilcox KS. Mechanisms of action of antiepileptic drugs. Int Rev Neurobiol 2007; 81: 85-110.

13. Nazıroğlu M, Yürekli VA. Effects of antiepileptic drugs on antioxidant and oxidant molecular pathways: focus on trace elements. Cell Mol Neurobiol 2013; 33: 589-99.

14. Sanchez-Villarejo MV, Lopez-Pedrajas R, Sanchez-Vallejo V, et al. Chronic cocaine effects in retinal metabolism and electrophysiology: treatment with topiramate. Curr Eye Res 2014; 39: 493-503.

15. Genc BO, Dogan EA, Dogan U, Genc E. Anthropometric indexes, insulin resistance, and serum leptin and lipid levels in women with cryptogenic epilepsy receiving topiramate treatment. J Clin Neurosci 2010; 17: 1256-9.

16. Minton GC, Miller AD, Bookstaver PB, Love BL. Topiramate: safety and efficacy of its use in the prevention and treatment of migraine. J Cent Nerv Syst Dis 2011; 3: 155-68.

17. Armagan A, Kutluhan S, Yilmaz M, et al. Topiramate and vitamin e modulate antioxidant enzyme activities, nitric oxide and lipid peroxidation levels in pentylenetetrazol-induced nephrotoxicity in rats. Basic Clin Pharmacol Toxicol 2008; 103: 166-70.

18. Cure MC, Cure E, Tumkaya $L$, et al. Topiramate has protective effect on renal injury. Bratisl Lek Listy 2015; 116: 259-63.

19. Naziroğlu M, Kutluhan S, Yilmaz M. Selenium and topiramate modulates brain microsomal oxidative stress values, $\mathrm{Ca}^{2+}$-ATPase activity, and EEG records in pentylentetrazol-induced seizures in rats. J Membr Biol 2008; 225: 39-49.

20. Erer D, Dursun AD, Oktar GL, et al. The effects of iloprost on lung injury induced by skeletal muscle ischemia-reperfusion. Bratisl Lek Listy 2014; 115: 405-10.

21. Lowry Oh, Rosebrough Nj, Farr Al, Randall Rj. Protein measurement with the Folin phenol reagent. J Biol Chem 1951; 193: 265-75.
22. Draper HH, Hadley M. Malondialdehyde determination as index of lipid peroxidation. Methods Enzymol 1990; 186: 421-31.

23. Kurt A, Tumkaya $L$, Yuce $S$, et al. The protective effect of infliximab against carbon tetrachloride-induced acute lung injury. Iran J Basic Med Sci 2016; 19: 685-91.

24. Kurt A, Tumkaya $L$, Kalkan $Y$, et al. Is adalimumab protective in ischemia-reperfusion injury in lung? Iran J Basic Med Sci 2015; 18: 1093-9.

25. Kurt A, Tumkaya L, Turut $H$, et al. Protective Effects of Infliximab on Lung Injury Induced by Methotrexate. Arch Bronconeumol 2015; 51: 551-7.

26. Pararajasingam R, Weight SC, Bell PR, Nicholson ML, Sayers RD. Pulmonary nitric oxide metabolism following infrarenal aortic cross-clamp-induced ischaemia-reperfusion injury. Eur J Vasc Endovasc Surg 2000; 19: 47-51.

27. Yip HK, Chang YC, Wallace CG, et al. Melatonin treatment improves adipose-derived mesenchymal stem cell therapy for acute lung ischemiareperfusion injury. J Pineal Res 2013; 54: 207-21.

28. Demirci $S$, Kutluhan $S$, Naziroğlu $M$, et al. Effects of selenium and topiramate on cytosolic $\mathrm{Ca}^{2+}$ influx and oxidative stress in neuronal PC12 cells. Neurochem Res 2013; 38: 90-7.

29. Baldus S, Heitzer T, Eiserich JP, et al. Myeloperoxidase enhances nitric oxide catabolism during myocardial ischemia and reperfusion. Free Radic Biol Med 2004; 37: 902-11.

30. Cardenas-Rodriguez N, Coballase-Urrutia E, Rivera-Espinosa L, et al. Modulation of antioxidant enzymatic activities by certain antiepileptic drugs (valproic acid, oxcarbazepine, and topiramate): evidence in humans and experimental models. Oxid Med Cell Longev 2013; 2013: 598493.

31. Cure E, Cure MC, Tumkaya L, et al. Topiramate ameliorates abdominal aorta cross-clamping induced liver injury in rats. Saudi J Gastroenterol 2014; 20: 297-303.

32. Cure E, Cumhur Cure M, Tumkaya L, et al. Adalimumab ameliorates abdominal aorta cross clamping which induced liver injury in rats. Biomed Res Int 2014; 2014: 907915.

33. Naidu BV, Woolley SM, Farivar AS, et al. Early tumor necrosis factor-alpha release from the pulmonary macrophage in lung ischemia reperfusion injury. J Thorac Cardiovasc Surg 2004; 127: 1502-8.

34. Mao X, Ji C, Sun C, et al. Topiramate attenuates cerebral ischemia/reperfusion injury in gerbils via activating GABAergic signaling and inhibiting astrogliosis. Neurochem Int 2012; 60: 39-46.

35. Chester $\mathrm{AH}$, Yacoub $\mathrm{MH}$. The role of endothelin-1 in pulmonary arterial hypertension. Glob Cardiol Sci Pract 2014; 2014: 62-78.

36. Gottlieb SS, Harris K, Todd J, et al. Prognostic significance of active and modified forms of endothelin 1 in patients with heart failure with reduced ejection fraction. Clin Biochem 2015; 48: 292-6.

37. Iwata $S$, Ito $S$, Iwaki $M$, et al. Regulation of endothelin-1-induced interleukin- 6 production by $\mathrm{Ca}^{2+}$ influx in human airway smooth muscle cells. Eur J Pharmacol 2009; 605: 15-22.

38. Kerem M, Bedirli A, Pasaoglu H, et al. Effect of adrenomedullin on hepatic damage in hepatic ischaemia/reperfusion injury in rats. Liver Int 2008; 28: $972-81$.

39. Guo X, Cao W, Yao J, et al. Cardioprotective effects of tilianin in rat myocardial ischemia-reperfusion injury. Mol Med Rep 2015; 11: 2227-33.

40. Xing D, Zhang R, Li S, et al. Pivotal role of mast cell carboxypeptidase A in mediating protection against small intestinal ischemia-reperfusion injury in rats after ischemic preconditioning. J Surg Res 2014; 192: 177-86.

41. Yang P, Yang N, Zhang X, Xu X. The significance and mechanism of propofol on treatment of ischemia reperfusion induced lung injury in rats. Cell Biochem Biophys 2014; 70: 1527-32.

42. Cheng XL, Li MK. Effect of topiramate on apoptosis-related protein expression of hippocampus in model rats with Alzheimers disease. Eur Rev Med Pharmacol Sci 2014; 18: 761-8. 